Preprint HU-TFT-92-1

8 January 1992

revised 9 March 1992

re-revised 22 April 1992

\title{
THE FREE ENERGY OF SPHERICAL BUBBLES IN LATTICE SU(3) GAUGE THEORY
}

\author{
K. Kajantie ${ }^{1}$, Leo Kärkkäinen ${ }^{2}$ and K. Rummukainen ${ }^{3}$
}

\begin{abstract}
We study the coefficients of the expansion $F(R)=\frac{1}{3} c_{3} R^{3}+\frac{1}{2} c_{2} R^{2}+c_{1} R$ of the free energy of spherical bubbles at $T=T_{c}$ in pure glue QCD using lattice Monte Carlo techniques. The coefficient $c_{3}$ vanishes at $T=T_{c}$ and our results suggest that the sign and the order of magnitude of $c_{1}$ is in agreement with the value $c_{1}= \pm 32 \pi T_{c}^{2} / 9$ (- for hadronic bubbles in quark phase, + for quark bubbles in hadronic phase) computed by Mardor and Svetitsky from the MIT bag model. The parameter $c_{2}$ is small in agreement with earlier determinations.
\end{abstract}

1) University of Helsinki, Department of Theoretical Physics, Siltavuorenpenger 20 C, 00170 Helsinki, Finland; kajantie@finuhcb.

2) Research Institute for Theoretical Physics, Siltavuorenpenger 20 C, 00170 Helsinki, Finland; permanent address Universität Bielefeld, Fakultät für Physik, Postfach 8640, D-4800 Bielefeld 1, BRD; leo@c240.uni-bielefeld.de.

3) CERN/TH, CH-1211 Geneve, Switzerland; rummukai@cernvm. 
Bulk QCD matter is stable in the quark-gluon plasma phase (Q) for $T \geq T_{c}$ and in the hadronic phase $(\mathrm{H})$ for $T \leq T_{c}$. At $T=T_{c}$ the free energies coincide and there is a latent heat $L$ :

$$
F_{q}\left(T_{c}\right)=-V p_{q}\left(T_{c}\right)=F_{h}\left(T_{c}\right)=-V p_{h}\left(T_{c}\right), \quad L=T_{c}\left[p_{q}^{\prime}\left(T_{c}\right)-p_{h}^{\prime}\left(T_{c}\right)\right]
$$

assuming the transition is of first order and taking the chemical potential $\mu=0$. The determination of the free energy has been studied in great detail with lattice Monte Carlo techniques [1]. At $T=T_{c}$ a stable $\mathrm{H}-\mathrm{Q}$ (order-disorder) interface can exist in the system and contributes to the free energy:

$$
F=-p V+\alpha A
$$

Lattice Monte Carlo computations [2-3] using planar interfaces have given the value

$$
\alpha\left(T_{c}\right) \approx 0.1 T_{c}^{3}
$$

for the interface tension in the case of pure glue.

The purpose of this letter is to go still one step further in the lattice Monte Carlo determination of free energies of domains of one phase immersed in the other: spherical bubbles of $\mathrm{H}$ phase in $\mathrm{Q}$ matter (or $\mathrm{Q}$ bubbles in $\mathrm{H}$ matter) [4]. Then the free energy of an $\mathrm{H}$ bubble (relative to a system entirely in $\mathrm{Q}$ phase) with radius $R$ can be expanded in powers of $1 / R$ as follows:

$$
F(T, R)=\left[p_{q}(T)-p_{h}(T)\right] \frac{4}{3} \pi R^{3}+\alpha(T) 4 \pi R^{2}+\gamma(T) R+\ldots
$$

The new element here is the last curvature term. Using the MIT bag model, i.e., treating the bubble as a spherical cavity, gluons as eight copies of Abelian photons, and assuming bag boundary conditions, Mardor and Svetitsky [4] found that

$$
\alpha_{\mathrm{bag}}(T)=0, \quad \gamma_{\mathrm{bag}}(T)=-\frac{32 \pi}{9} T^{2}
$$

for $\mathrm{Q}$ bubbles in $\mathrm{H}$ the sign of $\gamma$ is positive. Our main result is that also lattice Monte Carlo shows evidence of the pattern in eq. (5): a small area term $\alpha$ and a large curvature term $\gamma$, the numerical value of which is compatible with that in eq. (5). 
If only the terms shown in (4) contributed to $F(R)$, there would be an $R_{\text {min }}$ with $F\left(R_{\text {min }}\right)<F(0)=0$, i.e., $\mathrm{H}$ bubbles would be stable even for $T>T_{c}$. However, even in the bag model the terms in $1 / R$ omitted in eq. (4) decrease $F(0)-F\left(R_{\min }\right)$ and on the basis of our results presented here one cannot conclude anything on the stability of $\mathrm{H}$ bubbles for $T>T_{c}$.

Before presenting the lattice results, consider eq. (4) phenomenologically for $T \approx T_{c}$. Scaling with $T_{c}: \hat{T}=T / T_{c}, \hat{R}=T_{c} R$, we firstly can write $p_{q}(T)-p_{h}(T)=L(\hat{T}-1)$ and estimate the latent heat by $L=T_{c} p_{q}^{\prime}\left(T_{c}\right)=32 \pi^{2} T_{c}^{4} / 45$. For H-Q interfaces we do not know $\alpha(T)$ away from $T_{c}$, but a study of order-order interfaces in the $\mathrm{Q}$ phase [5] has given a very large value, $T \alpha^{\prime}(T) / \alpha(T) \approx 30$, for the surface entropy, $S_{s}=-\alpha^{\prime}(T) A$. We use this same value and write $\alpha(T) / T_{c}^{3}=0.1+3(\hat{T}-1)$. Our lattice data below indicates that $\gamma$ depends only weakly on $T$ and we finally have

$$
F(T, R) / T_{c}=4 \pi\left\{\frac{32 \pi^{2}}{135}(\hat{T}-1) \hat{R}^{3}+[0.1+3(\hat{T}-1)] \hat{R}^{2}-\frac{8}{9} \hat{R}\right\} .
$$

Quantitatively, this model implies that at $T=T_{c} \approx 200 \mathrm{MeV}$ a stable $\mathrm{H}$ bubble has a radius of about $4 / T_{c}=4 \mathrm{fm}$. The radius has shrunk to $1 \mathrm{fm}$ at $T=1.05 T_{c}$.

Our aim is to determine the coefficients of the expansion (4) at $T=T_{c}$. First, we point out that the expected magnitude of $\hat{\gamma}=\gamma / T_{c}^{2}=-32 \pi / 9$ is much larger than $\hat{\alpha}=\alpha / T_{c}^{3}=0.1$. This difference is essential for the success of our calculations: as it will turn out, the actual thickness of the bubble wall is of order $1 / T_{c}$, so that the definition of the radius of the bubble is necessarily ambiguous. For example, possible non-equivalent choices are the radius where the order parameter reaches some fixed value between the ordered and disordered states; the turning point of the radial order parameter; or the corresponding quantities for the energy density.

This ambiguity affects the functional form of $F(R)$. Let us redefine the radius $\hat{R} \rightarrow$ $\hat{R}_{1}=\hat{R}-\delta$, so that at $T=T_{c}$ :

$$
\hat{F}=4 \pi \hat{\alpha} \hat{R}^{2}+\hat{\gamma} \hat{R}=4 \pi \hat{\alpha} \hat{R}_{1}^{2}+(\hat{\gamma}+8 \pi \hat{\alpha} \delta) \hat{R}_{1}+\mathcal{O}\left(R_{1}^{0}\right)
$$

If now $\hat{\alpha}$ and $\hat{\gamma}$ are of the same magnitude, even a slight variation in $\delta$ will spoil the $\mathcal{O}\left(R_{1}\right)$ term. In fact, in this case a prime candidate for the bubble radius would be a 
value of $\mathrm{R}$ where $F=4 \pi \alpha R^{2}$ exactly! However, the values given above for $\hat{\gamma}$ and $\hat{\alpha}$ imply that $\delta$ would have to be $\approx 4 / T$ for the linear term to vanish. This is more than the wall thickness $W \sim 2 / T$, so that this $\delta$ would move the radius completely outside of the actual interface. Thus, the whole concept of the curvature contribution to the free energy makes sense only when $\gamma \gg 8 \pi \alpha W \approx 8 \pi \alpha / T$.

We would like to stress that this ambiguity in the subleading terms is of quite general nature; it is present both in physical phenomena and in lattice calculations. We shall return to this question more in detail further down.

On a homogeneous system at thermal equilibrium the bubbles are rare and lattice calculations would be extremely time-consuming. To remedy this and to gain systematic control of the bubbles, we choose a spatially spherical region of radius $R$ and adjust the couplings $\beta \equiv 2 N_{c} / g^{2}=\beta_{c} \pm \Delta \beta$ in the interior and exterior, respectively. This acts like an external field and stabilises the bubble. For simplicity, we call this $R$ the radius of the bubble, and check the sensitivity of the results to reparametrisations later. The coefficients of eq. (4) are now evaluated with various $\Delta \beta$, and the final answer is obtained by extrapolating $\Delta \beta \rightarrow 0$. In the calculations one cannot use too small a $\Delta \beta$, otherwise the bubble will vanish. We assume that the relevant quantities behave linearly in $\Delta \beta$, as long as the bubble is preserved.

It is not possibly to evaluate directly the free energy $F$ using a MC simulation. However, the derivatives of the free energy are accessible as ensemble averages, thus providing, after integration, the value of the free energy up to a constant. The 'standard' way to do this is to integrate with respect to the inverse coupling $\beta[1,2,3]$. However, as we are interested in the $R$-dependence of the free energy, it is natural to take the derivativee w.r.t. $R$, the bubble radius. With this method we avoid the very delicate and timeconsuming integration over $\beta$. However, in order to measure the derivative, we have to modify the action to form

$$
\sum_{\mathrm{n}} \beta\left(r_{\mathrm{n}}, R\right) S(n)=\sum_{\mathrm{n}}\left(\beta_{c}+\Delta \beta \tanh \frac{r_{\mathrm{n}}-R}{w}\right) S_{\mathrm{Wilson}}(n)
$$

where the sum is over the lattice, $r_{\mathrm{n}}$ is the distance from the center of the bubble and $w$ (chosen as 0.5 lattice spacings $a$ ) parametrises the width of the cross-over of the function $\beta(r, R) ; w$ is not to be mixed with the actual thickness of the interface. The quantity 
accessible by means of MC then is

$$
\frac{d(F / T)}{d R}=-\frac{\Delta \beta}{w}\left\langle\sum_{\mathrm{n}} \cosh ^{-2}\left(\frac{r_{\mathrm{n}}-R}{w}\right) S(n)\right\rangle,
$$

where the average is taken over the ensemble created with the action of eq. (8).

The role of the parameter $w$ deserves some elaboration. It was introduced to facilitate the evaluation of the derivative $d \beta\left(r_{\mathrm{n}}, R\right) / d R$ : the most obvious choice for $\beta(r, R)$ would be a step-like function $(w=0)$, but then eq. (9) becomes $-2 \Delta \beta \sum_{\mathrm{n}} \delta\left(r_{\mathrm{n}}-R\right) S(n)$. On a $3+1$ dimensional lattice this clearly puts different values of $R$ to a completely unequal footing, and the whole operator makes sense only when one integrates it over some range of $R$. In principle this requires separate simulations for each value of $R=r_{\mathrm{n}}$ in this range, which would be exceedingly costly. The form of $\beta(r, R)$ in eq. (8) smears the step over a shell $R-w \lesssim r_{\mathrm{n}} \lesssim R+w$. The larger $w$ is, the smoother one can expect the behavior of $F$ as a function of $R$ to be, and $F^{\prime}(R)$ measured at any single value of $R$ becomes meaningful. However, too large a $w$ does not pin down the physical surface well enough.

We chose $w=0.5 a$ in order to keep the cross-over in $\beta(r, R)$ within one lattice spacing, which is anyway the shortest length scale on the lattice. It turns out that this choice makes the function $F^{\prime}(R)$ sufficiently regular (although there still remains some systematic lattice effects, as seen from the similarity of the fluctuations of the data around the fits in Fig. 2.).

We stress that the use of the $w=0$ instead of non-zero $w$ does not make the calculations any more physical: when $\Delta \beta \rightarrow 0$, the explicit $w$-dependence of the action vanishes, and the configurations extrapolate to physical bubble configurations. One can also expect that the effects of a particular choice of $w$ become minimal. As discussed above, the bubble radius can be defined in numerous ways, and the discontinuity (or the cross-over) radius of the 'external field' $\Delta \beta(r, R)$ is not particularly good parametrisation, since it is not any intrinsic property of the bubble. For example, it will turn out that the radius where the order parameter reaches the value half-way between the values in the interior and exterior bulk states can deviate from the cross-over radius considerably more than the half-width of the cross-over itself (Fig. 1). This implies that even with finite $\Delta \beta$ the effects caused by choosing $w=0$ or $w=0.5$ are overshadowed by the difference between the 'intrinsic' radius and the 'external field' radius of the bubble. We remind 
again that this problem is only alleviated by the large value of the curvature term when compared to the interface tension.

On the lattice we actually measure $R$ in units of the lattice spacing $a=1 /\left(N_{t} T\right)$, and have, from eq. (4),

$$
\frac{d(F / T)}{R / a}=\frac{4 \pi}{N_{t}^{3}} \frac{p_{q}(T)-p_{h}(T)}{T^{4}} \frac{R^{2}}{a^{2}}+\frac{8 \pi}{N_{t}^{2}} \frac{\alpha(T)}{T^{3}} \frac{R}{a}+\frac{1}{N_{t}} \frac{\gamma(T)}{T^{2}}+\ldots
$$

Denoting the LHS by $F^{\prime}(R)$ we fit the lattice data to (from now on $R / a \rightarrow R$ )

$$
F^{\prime}(R)=c_{3} R^{2}+c_{2} R+c_{1}
$$

extrapolate the coefficients $c_{i}$ to $\Delta \beta=0$ and obtain the physical quantities at $T_{c}$ by comparison with eq. (10).

We have performed simulations on a $2 \times 16^{3}$ lattice using an algorithm for $\mathrm{SU}(3)$ gauge theory with a combination of pseudo-heat bath and overrelaxation sweeps. The values of $\beta$ were $\beta_{\text {in } / \text { out }}=5.1 \pm \Delta \beta$ with $\Delta \beta=0.5,0.25,0.125$ and the radius $\mathrm{R}$ varied between 2 and 7 in steps of 0.5 with some intermediate points in steps of 0.25 . The total number of iterations thus was 2 (for $\mathrm{H}, \mathrm{Q}) \times 47$ (for values of $\Delta \beta$ and $R$ ) $\times 10000$ (or sometimes 5000 ) iterations per point, amounting to a total of about $1 \mathrm{M}$ iterations or (with 2.7 s/sweep) about 700h of Cray XMP CPU time. By modern standard, the number of iterations on each point is relatively low, but the systems are rather far from the critical coupling and the autocorrelation times are small, $\lesssim 10$ sweeps.

The size of the lattice limits the physical bubble radius to $\leq 3.5 / T$. This is a serious limitation; it forces us to use relatively large $\Delta \beta$ to maintain the bubble on the lattice and to keep the correlation length small enough. Using eq. (6), one can see that at $T=T_{c}$ the expected stable radius is $4 / T_{c}$ and the radius where $F(R)=F(0)=0$ is $9 / T_{c}$. To truly study this region, the spatial size of the lattice should be at least $\approx 40 a$. The small size of the bubbles and large $\Delta \beta$ values can cause systematical errors, whose magnitude remains unknown; for example, the terms $\mathcal{O}\left(R^{n}\right), n \leq 0$ in eq. (4) can become significant. Also, for a more detailed analysis of the $\Delta \beta$ dependence one would need more than 3 values of $\Delta \beta$ for both $\mathrm{Q}$ and $\mathrm{H}$ bubbles.

Fig. 1 shows how the order parameter varies as a function of the distance from the center of the bubble for $\Delta \beta=0.25$; the pattern is similar for $\Delta \beta=0.5$ or 0.125 . One 
sees clearly the formation of an $\mathrm{H}$ bubble but with an interface thickness of some 4 units in $R$. In physical units the interface would be about $2 \mathrm{fm}$ thick: we have $1 / T_{c}=N_{t} a \approx$ $1 \mathrm{fm}$, so that $a \approx 0.5 \mathrm{fm}$. Remember that the input thickness $w$ was only half a lattice spacing.

The results for $F^{\prime}(R)$ and for the fitted parameters $c_{i}$ are shown in Figs. 2 and 3 for $\mathrm{H}$ bubbles in $\mathrm{Q}$ and in Figs. 4 and 5 for $\mathrm{Q}$ bubbles in $\mathrm{H}$.

Since the numerical values of $c_{3} R^{2}$ are rather large, we shown in Fig. 2 the measured values of $F^{\prime}(R)$ after subtracting the term $c_{3} R^{2}$ with the fitted values of $c_{3}$ shown in Fig. 3(a). Only values $R \geq 3$ are used in the fit. The linear behaviour of the data is evident, although the $\chi^{2} /$ d.o.f. is as large as 2-4 depending on the lower cutoff in $R$. We attribute the large value of $\chi^{2} /$ d.o.f. to a lattice discretisation effect: the number of plaquettes inside the bubble does not grow smoothly with increasing $R$.

The values of $c_{3}$ in Fig. 3(a) extrapolate linearly to $-0.38 \pm 0.10$ when $\Delta \beta \rightarrow 0$. Relative to the values extrapolated from, about $-80,-40,-20$, this is very close to the value 0 expected at $T=T_{c}$ (eq. (4)). Also the values for $\Delta \beta \neq 0$ are compatible with the pressure difference between homogeneous simulations at $\beta_{\text {out }}$ and $\beta_{\text {in }}$. Similarly, $c_{2}=$ $2 \pi \alpha / T^{3}$ (eq. (10)) extrapolates to the value $0.63 \pm 0.89$ when $\Delta \beta \rightarrow 0$. This coincides with the value (3) of $\alpha\left(T_{c}\right)$, but due to the large statistical errors we can confirm only the smallness of this value. Obviously, the methods utilised in [2,3] for planar interfaces are superior to the one used here for determining the interface tension $\alpha$. The values of $c_{2}$ for $\Delta \beta \neq 0$ are discussed below.

The main result is shown in Fig. 3(c): the curvature parameter $c_{1}=\gamma /\left(N_{t} T^{2}\right)$ as given by the intercept of the lines in Fig. 3(a) at $R=0$. It is independent of $\Delta \beta$ and well compatible with the bag model result. The errors are of the same magnitude as with $c_{2}$; however, due to the large value of $c_{1}$ the result is $3 \sigma$ away from zero.

The corresponding data and fits for $\mathrm{Q}$ bubbles in $\mathrm{H}$ presented in Figs. 4 and 5 show a similar average pattern, but the error bars in the fits are so large that they make the result only $1 \sigma$ away from zero. In spite of this, the central value of $c_{1}$ coincides with that obtained for $\mathrm{H}$ bubbles, but has an opposite sign. Due to the large errors in $\mathrm{Q}$ bubbles, in what follows we concentrate mainly on the $\mathrm{H}$ bubble case. 
From the derivative $F^{\prime}(R)$ we can obtain $F(R)$ by integration. The result is shown in Fig. 6, normalised arbitrarily to $F(0)=0$. One sees clearly the large numerical values for $\Delta \beta \neq 0$ caused by the dominant $R^{3}$ term. The distance of extrapolation to $\Delta \beta=0$ is evidently large and we have constrained $c_{3}(\Delta \beta=0)=0$. The resulting curve is in agreement with the phenomenological one in eq. (6). Notice, however, that one should not take the $1 / R$ expansion too seriously for very small $R$.

As discussed above, the definition of the physical radius of the bubble is necessarily ambiguous. It is essential to check the stability of the results against these reparametrisations. Let us perform a reparametrisation of $R$ consistent with the $R \rightarrow \infty$ behaviour:

$$
R \rightarrow R+x_{1}+x_{2} / R+\mathcal{O}\left(1 / R^{2}\right)
$$

Then

$$
F(R)=\frac{1}{3} c_{3} R^{3}+\frac{1}{2}\left(c_{2}+2 c_{3} x_{1}\right) R^{2}+\left[c_{1}+c_{2} x_{1}+c_{3}\left(x_{1}^{2}+x_{2}\right)\right] R+\mathcal{O}\left(R^{0}\right) .
$$

We are mainly interested in the term linear in $R$. In it $c_{3}$ is large but vanishes for $\Delta \beta \rightarrow 0$ and thus does not affect the physical value of $c_{1}$. In the remaining term we know $c_{2}$ from the value of the interface tension but $x_{1}$, which corresponds to a constant shift in the radius, remains a free parameter. Even if we adjust it by $\sim \pm 1$ lattice units, which should encompass all 'reasonable' radius redefinitions discussed above, the linear term in $R$ is only modified by $\sim 10 \%$. This is well below the statistical errors of $c_{1}$.

It is also possible to fix $x_{1}$ by comparing the second term with the values of the area term in planar interface simulations, i.e., the data in Fig. 3(b) with the data in Fig. 8 of [2]. One obtains $x_{1}=0.04$ independent of $\Delta \beta$, which shows the consistency of the discussion. Thus at $\Delta \beta=0$ the value of $c_{1} \approx-6$ is only modified by a term of the magnitude 0.03 ; totally negligible.

As discussed above, this seemingly surprising stability of $c_{1}$ is caused by the vanishing coefficient $c_{3}$ when $\Delta \beta \rightarrow 0$ and by the very small value of the interface tension $c_{2}$ when compared with $c_{1}$. For $\Delta \beta \neq 0$ the additional term $2 c_{3} x_{2}$ may explain why the data for $c_{1}$ seem independent of $\Delta \beta$ - clearly it is also possible that $\gamma(T) / T^{2}$ is only weakly dependent on $T$, as the bag model predicts.

In conclusion, our lattice Monte Carlo simulations support the existence of the curvature contribution $\gamma$ to the free energy of spherical bubbles predicted by the MIT bag model 
[4]. The value predicted by the bag model is $\gamma / T^{2}= \pm 32 \pi / 9$, which is the same order of magnitude than our results; although for the quark bubbles the result is not statistically significantly non-zero. However, one should emphasise that the lattice used has a small $N_{t}=2$, that even the smallest value of $\Delta \beta$ still is quite large, that the bubble radii accessible to us may be too small to justify the truncation of the $1 / R$-expansion used here, and that there is no control of finite $V$ effects. It is quite conceivable that more refined analyses will lead to a different numerical value, but we nevertheless find it quite interesting that Monte Carlo computations give such a clear signal for the MIT bag model value for the curvature term. For the interface tension $\alpha$ we could not obtain a statistically significant result. With further Monte Carlo runs one should also be able to make statements concerning $F(R)$ when $R \rightarrow 0$ and to address the question of stability of $\mathrm{H}$ bubbles for $T>T_{c}$ quantitatively.

\section{Acknowledgements}

We thank the Finnish Center for Scientific Computing for computing facilities, the Academy of Finland for financial support and Jean Potvin, Ben Svetitsky and Frithjof Karsch for discussions and comments. 


\section{References}

[1] J. Engels, J. Fingberg, F. Karsch, D. Miller and M. Weber, Phys. Lett. B252(1990) 625.

[2] K. Kajantie, Leo Kärkkäinen and K. Rummukainen, Nucl. Phys. B333 (1990) 100.

[3] S. Huang, J. Potvin, C. Rebbi and S. Sanielevici, Phys. Rev. D42 (1990) 2864.

[4] Israel Mardor and Ben Svetitsky, Phys. Rev. D44(1991)878.

[5] K. Kajantie, Leo Kärkkäinen and K. Rummukainen, Nucl. Phys. B357(1991)693. 


\section{Figure Captions}

Fig. 1. The behaviour of the order parameter, the Wilson line $L$, as a function of the distance $r$ from the center of the $\mathrm{H}$ bubble for $\Delta \beta=0.25$ and for $R=3,4,5,6,7$. The dotted line shows $\beta(r, R=5)$.

Fig. 2. $F^{\prime}(R)-c_{3} R^{2}$ for $\mathrm{H}$ bubbles. From up to down, $\Delta \beta=0.5,0.25,0.125$; the straight lines are the fits $c_{2} R+c_{1}$.

Fig. 3. The fitted values of (a) $c_{3}$, (b) $c_{2}$, (c) $c_{1}$ as a function of $\Delta \beta$ for $\mathrm{H}$ bubbles with a linear fit to the data points and an extrapolation to $\Delta \beta=0$. In (c) the arrow shows the bag model point $-16 \pi / 9$.

Fig. 4. $F^{\prime}(R)-c_{3} R^{2}$ for $\mathrm{Q}$ bubbles. From up to down, $\Delta \beta=0.5,0.25,0.125$; the straight lines are the fits $c_{2} R+c_{1}$.

Fig. 5. The fitted values of (a) $c_{3}$, (b) $c_{2}$, (c) $c_{1}$ as a function of $\Delta \beta$ for Q bubbles with a linear fit to the data points and an extrapolation to $\Delta \beta=0$. In (c) the arrow shows the bag model point $16 \pi / 9$.

Fig. 6. The free energy $F(R) / T$ at $T=T_{c}$, normalised to $F(0)=0$, integrated from the measured values of $F^{\prime}(R) . R$ is in units of $a$ and the curves are from below for $\Delta \beta=0.5,0.25,0.125$ and for the extrapolation to $\Delta \beta=0$. 